\title{
COSMOLOGY = TOPOLOGY/GEOMETRY: MATHEMATICAL EVIDENCE FOR THE HOLOGRAPHIC PRINCIPLE
}

\author{
by Adel N. Alahmadi and David G. Glynn
}

(with six text figures)

\begin{abstract}
Alahmadi, A.N. \& Glynn, D.G. 2016 (31:viii) Cosmology = topology/geometry: mathematical evidence for the Holographic Principle. Papers and Proceedings of the Royal Society of Tasmania 150(1): 31-38. https://doi.org/10.26749/rstpp.150.1.31 ISSN 0080-4703. King Abdulaziz University, Jeddah, Saudi Arabia (ANA, DGG); CSEM, Flinders University, Adelaide, SA 5001, Australia $\left(\mathrm{DGG}^{*}\right) .{ }^{*}$ Author for correspondence. Email: david.glynn@flinders.edu.au
\end{abstract}

\begin{abstract}
In August 2015 NORDITA (Nordic Institute for Theoretical Physics) hosted a conference where Hawking strongly supported the conjectured relationship between string theory and quantum fields that was initiated with the holographic principle some 20 years ago by 't Hooft, Maldacena, Susskind and Witten. We bring together results of several papers showing how mathematics can come to the party: the fundamentals of flat (even higher-dimensional) space can be derived very simply from topological properties on a surface. Specifically, Desargues, Pappus or other configurations do not have to be assumed a priori or as self-evident (a fundamental weakness of Hilbert's work in 1899) to develop the foundations of geometry. Are black holes places where non-commutative (quantum) behaviour reigns while Euclidean (flat) space is where commutativity holds sway? So, we cannot hope to look inside a black hole unless we know how "deformable" topology is related to "flat" geometry.
\end{abstract}

Key Words: cosmology, Desargues theorem, geometrical configuration, graph, history of mathematics, holographic principle, Pappus theorem, projective geometry, quantum mechanics, topological surface.

\section{INTRODUCTION}

Cosmology is about understanding the universe (Hawking 1988, Baker 2014). Many questions arise (Feynman 1992): what is space-time, how are particles related by forces? Start with the problem of space. Maybe it is Euclidean: flat or linear? It certainly looks superficially like that. The most recent advances point to a solution boiling down to holographic duality that must exist due to certain quantum constraints (Susskind 1995, 't Hooft 2000, 2009, Overby 2013, Cowen 2013). "Holographic" just means the conversion of a higher dimension to a lower one, or vice-versa, while "duality" has properties changing to analogous ones in the other. Thus the AdS/CFT or Maldacena duality (Maldacena 1998, 2013, Witten 1998) has been very useful in high-energy physics (Hamma \& Markopoulou 2011). The IT from Qubit project based at Stanford University (IT from Qubit 2015), a collaboration funded by the Simons foundation (the Chern-Simons gauge theory is well-known) put forward a concise list of problems, in some sense related to the holographic principle with the "bulk", that is, what we perceive to be space, being (intuitively) surrounded by the lower-dimensional "boundary". Of course, geometers have had a similar idea for centuries: ordinary (affine) space has "points at infinity" which form a hyperplane, and together they form a far more symmetric structure, projective space. But the physicists are going one step further: they say that properties of the boundary are much more closely linked with the properties of the bulk.

The classical duality of projective geometry goes to the projective dual by a change of language: points change to hyperplanes and so on. There are many other transformations called "dualities". For example, "matroid theory" converts edges of graphs (vertices are connected by edges) to points in space (over any given commutative field of coordinates). Here we describe a fundamental duality, found in recent papers by the authors, relating topology and projective geometry: it may have applications in cosmology because the foundations of geometry form the prerequisite for cosmology.

Geometrical development in a nutshell is as follows: after Euclid in about 300 BCE, contributions by Pappus (320 CE), see the translation by Ver Eecke (1933), and Desargues, an associate of Descartes (Desargues 1639, Bosse 1648, Field \& Gray 1987), non-Euclidean geometry arrived in the nineteenth century CE. In the beginning this involved Gauss (unpublished work) and the mathematicians Bolyai and Lobachevsky.

Thus one of the main and fundamental areas of geometry, projective geometry, was developed, first by the French (Carnot 1803) and then by the English, Germans and Italians in the nineteenth century. The Frenchman Poncelet wrote notes on geometry in a Russian military prison from 1812-1814, followed by the Germans Möbius (1828), Grassmann the elder (1844) and Karl Von Staudt (1847), the nobleman from Rothenburg ob der Tauber (see Plaumann \& Strambach (1981) for Staudt's innovative point of view). Following this tradition came Felix Klein and his "Erlangen Program" (Klein 2004). This program says that groups of symmetries are equivalent to the various kinds of geometry. It had all kinds of developments, including the use of group representations in physics to represent particles. In Klein (1926-27) there is also a valuable history. Most groups involved with physics are "Lie" or continuous groups; Sophus Lie was a Norwegian associate of Klein. In the twentieth century the geometry was generalised, with technical details such as linear dependence properties being better explained by Whitney's theory of matroids. Late in the nineteenth century, prompted by the foundational work of the Englishman Cayley and the German Riemann (1854), Klein, Poincaré, Minkowski, then Einstein incorporated higher-dimensionality to explain space-time properties like relativity and gravity. The speed of light is basically constant, and space is deformed by mass, a property that can be explained by assuming the equivalence of spatial 
frames of reference (Feynman 1992). Some works devoted to the foundation of geometry are: Russell (1897), Hilbert (1902) and Robinson (1940).

Theories such as M-theory and the standard model of particle physics suppose many dimensions of geometrical space: particles and forces may be strings vibrating according to some representations of groups; see Strogatz (2003), Connes (2004) and Gefter (2014a, 2014b). Dark matter seems to interact only very weakly with our visible world, giving unsolved problems, but is thought by some theorists to affect our space, and gravity, via other dimensions. However, most physicists rely at the basic level on properties of straight lines, flat planes, real number systems, all deriving from Euclidean roots and traditions. Klein (1926-27) knew that non-Euclidean geometry is just usual flat geometry with a change of metric given by a quadratic form.

Glynn (2013) and Alahmadi \& Glynn (unpublished) have shown that the basic properties of linear geometry are derived from low-dimensional topology. Specifically, on well-known topological surfaces (with very important holes), there are two-dimensional structures, maps, that are continuously deformable, preserving the properties of points and arcs joining them, and regions confined by these arcs: we can say that these should lead to the most basic linear geometry. Anyone can now use topology to construct the linear configurations that were shown to be fundamental. Furthermore, the topological counterparts are simpler and more general in many cases.

Thus it does look like, from the mathematical point of view, that space itself is like a hologram: a kind of higherdimensional illusion formed by properties that are really only two-dimensional, no matter how many dimensions we appear to detect with our senses or advanced physical devices. Sometimes art precedes science: Picasso already had realised that art is made from illusions. Cubism and unusual works forced people to think about reality (Gombrich 1977). It is probably no coincidence that Cubism developed around the same time as Einstein's revolution in physics: the first decade of the 1900s.

In the past, physicists such as Feynman have had some doubts when it comes to extending linear geometry down to microscopic quantum (or Planck) scales:

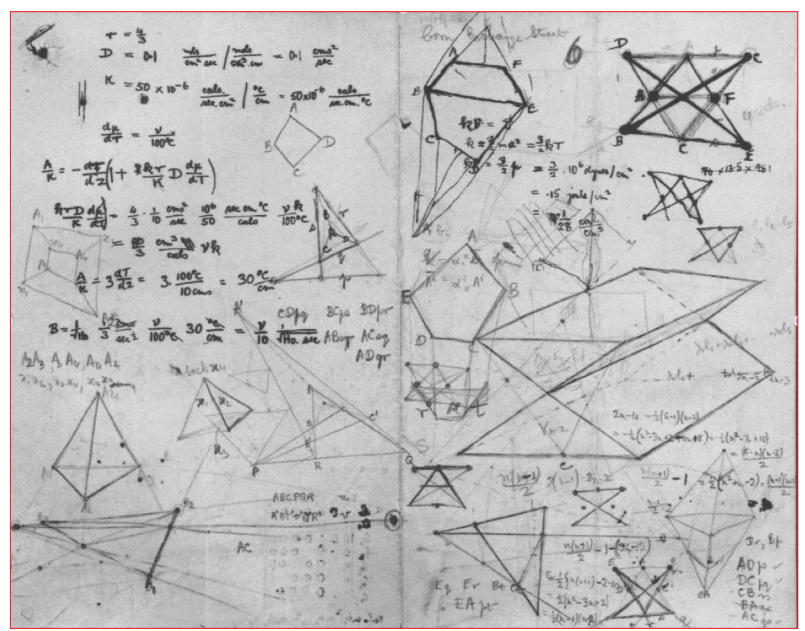

FIG. 1 - Paul Dirac, sketches.
The theory that space is continuous is wrong, because we get these infinities and other difficulties, and we are left with questions on what determines the size of all the particles. I rather suspect that the simple ideas of geometry, extended down into infinitely small space, are wrong. Here, of course, I am only making a hole, and not telling you what to substitute. If I did, I should finish this lecture with a new law. (Feynman 1992, pp. 166-167)

But Dirac, one of the founders of quantum mechanics, relied on geometric foundations, in particular projective geometry, to obtain his theories (Farmelo 2005, Galison 2000). This led to his joint Nobel prize with Schrödinger in 1933. In the upper right of figure 1 we see Dirac has drawn the 9 point 9 line Pappus configuration, "fundamental" for geometers. We shall see how to obtain this theorem in projective geometry using the duality with topology (in several ways). Pappus is known as the main property of space related to the multiplicative commutativity $(x y=y x)$ of the field of coordinates.

Heisenberg, when it came to the important noncommutativity, $A \times B \neq B \times A$ of observables in quantum physics, as compared with the more intuitive commutativity $A \times B=B \times A$ of Newtonian physics, was at first surprised (Galison 2000). But we shall see that non-commutativity (of the coordinate field) comes naturally from planarity of graphs. Might it be that it is just areas on a fundamental (or "cosmic") surface that have no holes that are quantum? We expect our new duality to support the physics.

\section{IDEAS FROM MATHEMATICS}

Here we recall some basic topology, graph theory, projective geometry and matroid theory, giving a quick history and some references.

\section{The topology of orientable surfaces}

This is quite a modern area that has infiltrated diverse areas from deep algebraic geometry with the study of algebraic curves, to the combinatorics of graphs. The origins of topology were with Euler (1741) and Listing (1847), see also

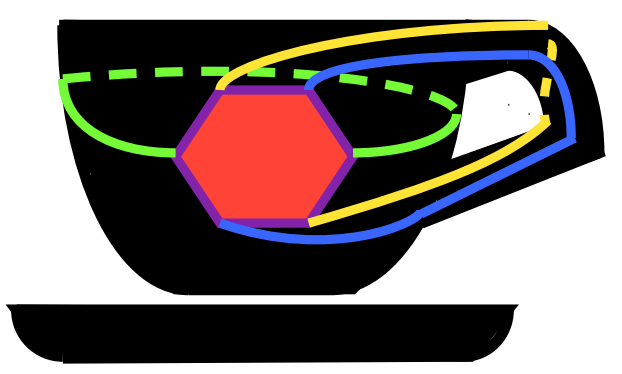

FIG. 2 - Geometry on a coffee cup: the toroidal graph $\mathrm{K}_{3,3}$ with six vertices, nine edges and three hexagonal faces. The vertices are those on the central red hexagon, which has six purple edges. There are three further edges that use the handle to avoid each other. The two other faces each have three purple edges which alternate with the green, the blue and the yellow. 
Tait (1883), and Poincaré (1895). We need some theory of graphs on surfaces, and more details, also with connections to physics, which can be found in Mohar \& Thomassen (2001), Franzosi \& Guadagnini (1996), Nash \& Sen (1983) and Stewart (1987).

We only need to know about closed oriented or orientable surfaces. (Compact Riemann surfaces have additional (complex) structure but we don't use these.) The surface of a ball, a doughnut, a coffee cup (fig. 2), are all archetypal examples of closed orientable surfaces. We contemplate the surface of such an object: it has no clear boundary curves it is "closed" - and locally within a limited region, it could as well be flat. But globally, or looking from an external viewpoint, the ball and the doughnut are different, and the important difference is measured by the number of holes it has: the "genus". Then the doughnut and the coffee cup are topologically the same. For a sphere the genus is zero, whereas for a doughnut, mathematicians call it a "torus", the genus is one. Here, we don't talk about spheres or doughnuts, but we refer to them as surfaces of genus 0 or 1 . The orientable nature of the surface comes in when we draw a small circle around any point on the surface. Then the circle can be given a natural cyclic order (clockwise or anticlockwise). If the surface is "connected", which we always assume, in a continuous move, without jumping at any stage from the surface, one can go to any other point, and the cyclic ordering on the surface is consistent: it cannot change from clockwise to anticlockwise in a continuous manner. Given an "orientable" surface, choosing one oriented direction, e.g., clockwise, at each of its points, makes it "oriented". What this also means in practice, is that the surface is "two-sided". Think about the sphere for example. There is an inside surface and an outside surface. A small clockwise circle on the outside induces a small anticlockwise circle on the inside, and viceversa. So the outside and the inside are separated topologically. There are also closed non-orientable surfaces: a change of the German umlaut to an "s" changes "kleinsche Fläche" (Klein's surface) into "kleinsche Flasche" (Klein's bottle); and there are non-orientable surfaces with boundary, such as the Möbius strip ("Band" in German), actually first published by Listing (1847), but these are not of major importance here. Linear geometry appears to be connected with certain properties of closed orientable surfaces and we can't yet see a connection with the other types of surfaces.

\section{Graphs and maps on orientable surfaces}

A graph (Tutte 1959) is a finite collection of points (called vertices), and a finite collection of "edges", or for physicists perhaps "strings" between the points. Usually, these edges are defined by unordered pairs or subsets $\{\mathrm{X}, \mathrm{Y}\}$ of two distinct vertices (if the graph is "simple"). The direction of the edges, from $\mathrm{X}$ to $\mathrm{Y}$ or from $\mathrm{Y}$ to $\mathrm{X}$ does not matter. (Non-simple) graphs with more than one edge between two vertices are said to have "multiple" edges.

Now a general graph may be embedded on a surface: the vertices become points on the surface, and the edges are arcs that do not meet on the surface except at their end-points. Any collection of arcs going around in a circuit and finishing at the same place will enclose a certain area on the surface, and if the surface is orientable, the surface will be cut into several pieces. In particular, if the circuit is minimal and contains none of the holes in the surface, then the interior of the circuit is a region or face of a certain map: we say that the face is "contractible" continuously to a point. The interiors of all the faces of the map will cover all the points of the surface, all except for the vertices of the graph and its edges. Each edge will lie on the boundary of two faces (except for some degenerate situations).

The number of holes in an orientable surface is called its genus $g$, which is a "homotopy" invariant: that is, two orientable surfaces of the same genus are deformable to each other. They have the same combinatorial properties, and it is these properties that we need.

It is a fundamental fact that if we have a graph having $v$ vertices, $e$ edges, and $f$ faces on an orientable surface of genus $g$, then $v-e+f=2-2 g=\chi$, the Euler-Poincaré characteristic, which relates $v, e, f, g$ by a linear equation.

For example, the graph $\mathrm{K}_{4}$, the complete graph with four vertices and having all possible six edges, is clearly embeddable on the surface of a sphere in Euclidean space: it is a tetrahedron, and then there are four faces, so that $v=f=4$, and $e=6$, satisfies $v-e+f=2$. So it checks out that $g=0$ on a sphere.

\section{Projective geometry}

For the modern foundations of projective geometry one looks to Hilbert (1902). Soon after this appeared Veblen \& Young $(1910,1917)$ produced simple axioms for projective geometry. Thus a projective space $S=(\mathrm{P}, \mathrm{L}, \mathrm{I})$ is assumed to be a set of points $\mathrm{P}$, with a set $\mathrm{L}$ of subsets of $\mathrm{P}$ called "lines". If a point $p$ is in (or "on") a line $r$ we say that the point and line are "incident": this defines the incidence relation $p \mathrm{I} r$. Points on the same line are said to be "collinear". Then there are some axioms (Dembowski 1968, pp. 45-46):

1. Every line has at least three points (Fano axiom).

2. Any pair of distinct points $p, q$ are contained in a unique line denoted by $p q$.

3. For any "triangle" $a, b, c$ of distinct non-collinear points, any pair of lines not through $a$ that intersect the lines $a b$ and $a c$ non-trivially will have a common point (Veblen axiom).

A "subspace" of $S$ is a subset of points, closed under the operation of joining points together to form lines, i.e., it is a set $T$ of points such that $p, q \in T$ implies $p q \subseteq T$. The set of all subspaces of $S$ is defined to be the "projective geometry" based on S. The subspaces, increasing in size, are the empty set $\varnothing$, the points (singleton sets), the lines, the planes (generated by triangles), and so on up to the hyperplanes, and the whole space. The "rank" of a subspace is the size of a minimal set of points that it has, generating the whole subspace, and then the "dimension" is the rank minus one. For any pair of subspaces $U, V$ there is an important dimension formula, (often called Grassmann's formula): $\operatorname{dim} U+\operatorname{dim} V=\operatorname{dim} U V+\operatorname{dim} U \cap V$. Here $U V$ is the smallest subspace containing $U$ and $V$; and $U \cap V$ is always a subspace.

Projective geometry of dimension more than one can be divided into three basic camps:

1. Projective (pappian) geometry over a field $F$ that is commutative, e.g. the real numbers $R$ or the complex numbers $C$;

2. Projective (desarguesian) geometry over a field $F$ that is non-commutative (called "division ring" or "skewfield", e.g., Hamilton's quaternions $H$ );

3. General projective planes (of dimension two), not usually having any base field, but instead more general coordinates such as over a quasifield, semifield or nearfield (Dembowski 1968). 
For us, the interest lies in projective geometry (1) and (2). Even though (3) has great theoretical interest we ignore this possibility from now on, since it is well known that a projective geometry of dimension at least three must be one of the first two types. A proof uses Desargues and Pappus ctheorems (see Veblen \& Young 1910, 1917). Thus we assume that the most general projective geometries are over a field where we don't know if it is commutative or not: Desargues ctheorem (see later) is an example of a property of space that holds in the most general projective geometries, but Pappus theorem would hold only for "commutative" projective geometries.

An affine space, or geometry, can be easily embedded into a projective geometry by adjoining a unique hyperplane at infinity. This is just another set of points that are defined by the parallel classes of lines. Conversely, any affine geometry (such as the Euclidean one), can be constructed from the projective geometry by deleting the hyperplane at infinity. Usually it is better for foundational work to use the projective geometry, since the axioms are much simpler. In general, in terms of collineation groups (Klein's Erlangen Program point of view), projective geometry corresponds to the transformations by non-singular matrices, while affine geometry corresponds to more complicated affine transformations.

We let PG $(n, F)$ be the projective geometry of dimension $n$ over a field $F$. It can be shown to be unique, subject to the proviso that if the field is non-commutative there could be two projective geometries related by anti-isomorphism, switching left and right, but it really is not important.

The easiest way to learn about it is to first understand the mathematical theory of fields $F$, and then go through the axioms of a vector space $\mathrm{V}=\mathrm{F}^{n+1}$ of dimension $n+1$ over $F$. If $F$ is non-commutative one has to choose either a left or right vector space. Then the projective geometry over $F$ of dimension $n$ is just the set of all vector subspaces of $V$. A vector subspace of rank $i+1$ corresponds to a projective subspace of dimension $i$. The language of vectors also changes to geometrical language. The vector subspace $\{0\}$ is just the empty set of points;

- a vector subspace of all multiples of a vector is just a point;

- a vector subspace generated by two independent vectors is just a line;

- a plane is generated by three independent vectors;

- a hyperplane is a vector subspace of generated by $n$ independent vectors;

- and $n+1$ vectors generate the whole space which is the same as the projective geometry $\operatorname{PG}(n, F)$.

Of course, one may dispense with algebra and choose to define projective geometry from a few lines of simple axioms that we gave above, but in practice, one needs the backup of algebraic coordinates (mostly "homogeneous" or "projective" coordinates) to solve many problems. A big part of it, though, is psychological: people are reassured when they use coordinates or vector spaces to construct geometry. It is like having nuts and bolts instead of abstract notions and axioms. However, nuts and bolts can also weigh one down: often things are more efficient if unnecessary items are discarded.

\section{Matroid theory}

This is a more recent theory developed by Hassler Whitney (1935), followed by W.T. Tutte starting in the late 1940s; see
Tutte (1959). It has been shown to be critical for a proper understanding of abstract ideas in geometry involving linear dependence, i.e., the concepts of basis, spanning set, circuit etc. Although we do not need the theory directly here, there are parallels with the idea of duality: e.g., each graph (not necessarily topological), corresponds to a circuit matroid, which is a configuration in geometrical space. A planar graph has a dual (switching vertices and faces) which gives the dual matroid, which, algebraically, comes from a kind of orthogonal space.

\section{FUNDAMENTAL THEOREMS}

Let $S$ be an orientable (two-sided) surface of genus $g$. This could well be associated as in the holographic principle with a "cosmic" surface that is the boundary of the "bulk": the CFT (lower-dimensional) side of the Maldacena duality. Let $G$ be a graph with possible multiple edges that is embedded on $S$. It has a map $M(G)$ with $v$ vertices, $e$ edges, and $f$ faces, so that the Euler characteristic equation is $v-e+f=2-2 g$. From the orientability one can put a clockwise orientation of the edges around each vertex of $G$, which induces an anticlockwise orientation around the inside of the face, and clockwise on the outside.

We give the two main theorems without proofs, which use topological reductions, and special kinds of determinants, some of which are valid also for non-commutative fields. The two theorems have different methods to produce "ctheorems" of slightly different types from the graph $G$. Abstractly, a ctheorem is a set $D$ of subsets of a finite set $C$, such that when the elements of $C$ are considered to be points in a given projective space, and when each subset in $D$ is now a (linearly) dependent set of the embedding, then all but one of the dependencies imply the remaining one. Recall that a "dependent" set has the (matroid) property that the subspace generated by some proper subset encloses the entire set. Thus ctheorems are special kinds of geometrical configuration, which describe the properties of linear space. Alahmadi \& Glynn (unpublished) have shown that the two main theorems produce ctheorems from the same (embedded) graph $G$ which are projectively dual when looked at in certain way: the two main theorems here are giving two sides of the same coin. However, direct methods to prove one theorem from the other, and other connections, could be explored further.

Most importantly, the ctheorems that either of the Theorems 1 and 2 produce are valid for the projective geometries of both types (1) and (2) over the most general division rings $F$ if the surface $S$ has genus $g=0$. That is, $G$ is a planar graph and the surface has no holes. The ctheorems extracted from either of the Theorems 1 and 2 using surfaces with at least one hole are valid for all projective geometry of type (1) over commutative fields $F$. This provides an explanation (for perhaps the first time) for the major dichotomy in projective geometry between commutative and non-commutative fields.

\section{Theorem 1 (Glynn 2013)}

The graph $G$ gives rise to a geometric property of general projective space. This is a configurational theorem (we call it more briefly a "ctheorem") having $v+f$ points and $e$ subsets (dependencies) of $v$ points, each in a hyperplane of PG $(v-1, F)$. For any embedding of the ctheorem, if $e-1$ of the hyperplanes are known then in general the final hyperplane also exists. 
Construction of the ctheorem:

- The vertices and faces become the points of the configuration.

- Each edge becomes a hyperplane containing the $v-2$ vertices not on the edge, and also the two faces of which the edge is a boundary.

Note: There is a (topological or matroid) dual ctheorem which switches the roles of vertices and faces, and so has $v+f$ points and $e$ hyperplanes in $\operatorname{PG}(f-1, F)$. Using projective duality there are also ctheorems with $e$ points and $v+f$ hyperplanes in $\operatorname{PG}(v-1, F)$ or $\operatorname{PG}(f-1, F)$.

\section{Theorem 2 (Alahmadi \& Glynn unpublished)}

The graph $G$ gives another ctheorem in $\operatorname{PG}(n, F)$, where $\operatorname{Max}(G) \leq n+1 \leq v,(\operatorname{Max}(G)$ is the size of the largest face of $G)$, having $v+e$ points and $e+f$ dependencies.

Construction of the ctheorem:

- The vertices and edges become the points of the configuration.

- Each edge gives a dependent line of three points, the two end vertices of the edge and the edge itself. (It follows that if there are $m$ multiple edges between two vertices all the corresponding $m+2$ points in the ctheorem are collinear.)

- Each face of $G$ gives a dependent set of edges, those edges on the boundary of the face. See figure 3 .

Notes:

- As in Theorem 1, Theorem 2 could use the (topological) dual graph of $G$ on the surface (changing around vertices and faces, or $v$ and $f$ ) giving another ctheorem. However, in this case the ctheorem is not the matroid dual.

- The bound for the dimension $n$ of the space in Theorem 2 is to prevent degeneracy.

- The Theorem 2 construction is related to (but not the same as) the classical way to get a configuration from a graph in matroid theory: for the circuit matroid of a graph one maps edges to points in projective space PG $(v-1, F)$ ( $F$ commutative), and circuits in the graph to minimal dependencies. However, Theorem 2 is clearly quite different, since the circuit matroid of a graph is almost never a ctheorem, and it is not defined for noncommutative fields.

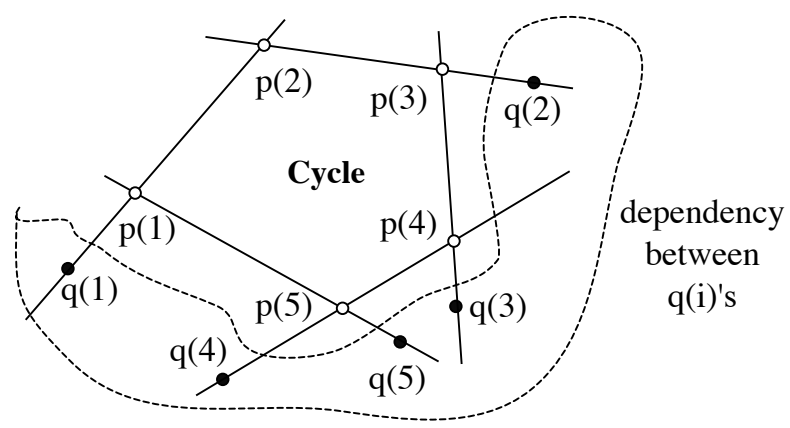

FIG. 3 - Cycle dependency (p's are on the boundary surface $S$ and q's are bulk points).

\section{EXAMPLES}

Hilbert (1902) showed that the properties of projective geometries (1) can be built up starting with Pappus ctheorem (fig. 6), and for projective geometries (2) with Desargues ctheorem (fig. 4). Both Pappus and Desargues are planar (two-dimensional) ctheorems of types $n_{3}$, where $n=9$ and 10 respectively, with $n$ points, $n$ lines, three points on each line, three lines through every point.

Hessenberg (1905) showed how to prove Desargues synthetically (using no algebra) given that Pappus holds: this is equivalent to the fact that the commutative fields are more specialised than non-commutative ones. For our duality it is the condition that a graph be planar.

\section{Desargues ctheorem (Desargues 1639, Bosse 1648)}

The complete graph on $v=4$ vertices, $\mathrm{K}_{4}$, is planar, having $e=6$ edges and $f=4$ triangular faces in its embedding. The orientable surface has genus zero and so Theorem 2 implies a configurational theorem having $v+e=10$ points, $e=6$ line dependencies and an additional $f=4$ face dependencies, which in this case are lines. Notice also that it is a theorem in both 2 - and 3 - dimensional space, since 3 is the minimum face size of $\mathrm{K}_{4}$, and there are 4 vertices in the graph. Since the surface has genus zero, the theorem from $\mathrm{K}_{4}$ is valid for all projective spaces over fields or skewfields. It is Desargues ctheorem.

In fig. 4 one can check that each triangular face of $\mathrm{K}_{4}$ corresponds to a unique dotted line. Thus in total there are 10 lines, six from the edges of $K_{4}$, and four dotted lines from the faces. Notice that the outside face must be included.

It is to be noted that if one used Theorem 1 instead of Theorem 2, the bundle theorem of 3- space would be obtained. Hence there are two ctheorems, in some way related by projective duality, coming from the same planar graph.

\section{Pappus (hexagon) ctheorem (Pappus 320 CE)}

The graph needed by Theorem 2 is a topological dual of the nonplanar graph $\mathrm{K}_{3,3}$ (the complete bipartite cubic graph having six vertices), embedded on the torus, of genus $g=1$,

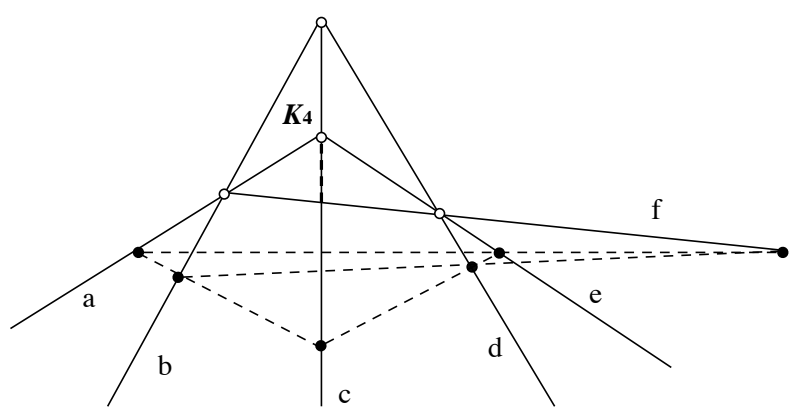

FIG. 4 - Complete planar graph $\mathrm{K}_{4}$, using Theorem 2, implies Desargues ctheorem. 
drawn in figure 2. That graph had three hexagonal faces, six vertices, and nine edges, so the dual needed (by flipping vertices and faces) for Pappus has three vertices, nine edges and six faces. First we have to draw it in another way.

There are several ways of thinking about a torus. The classical way is to consider any parallelogram in the Euclidean plane, and let it generate a tessellation covering all the points of the plane. Then it is clear that modulo this parallelogram, the opposite sides are identified with each other in the obvious way. It turns out that then the points inside and on the boundary (with the given identification of opposite sides) is the torus.

However, we shall use a slightly different, but actually equivalent method to represent the torus. Consider a regular hexagon in the same Euclidean plane. It also corresponds to a tessellation (or tiling) of all the points. Similarly, we identify the opposite sides of the hexagon, and it turns out that the points on the hexagon again form a torus. The three alternating points on the boundary of the hexagon correspond to the same point on the torus. Then we can split the hexagon into six triangles all touching at the central point as in figure 5.

This gives the graph $3 \mathrm{C}_{3}$ (the 3 -cycle with each edge repeated thrice) with three vertices, $X, Y$ and $Z$, and nine edges $a, b, c, d, e, f, g, h, i$. Thus we are basically tiling the hexagons over the Euclidean plane, and this map on the torus corresponds to a tesselation into triangles that are inside the tesselation of larger hexagons. Bees already know of the most efficient way to cover the plane! (Hales (2001) proved this.)

Theorem 2 shows that the lines of Pappus (displayed in fig. 6) are of two types: coming from triples of edges between the same two vertices, e.g., XY gives the edges $a$, $b, c$; and $b, d, g$ comes from a face.

Pappus lies in two-dimensional space: the graph has three vertices, so an upper bound for the dimension is two; and the largest face of the map induced by $3 \mathrm{C}_{3}$ is also three; so a lower bound for the dimension is two. Thus Pappus is a ctheorem for 2- $\mathrm{d}$ space, the projective plane. The plane must also be coordinatised by a commutative field, since the genus of the torus is one, which is greater than zero (one hole is there in the surface).

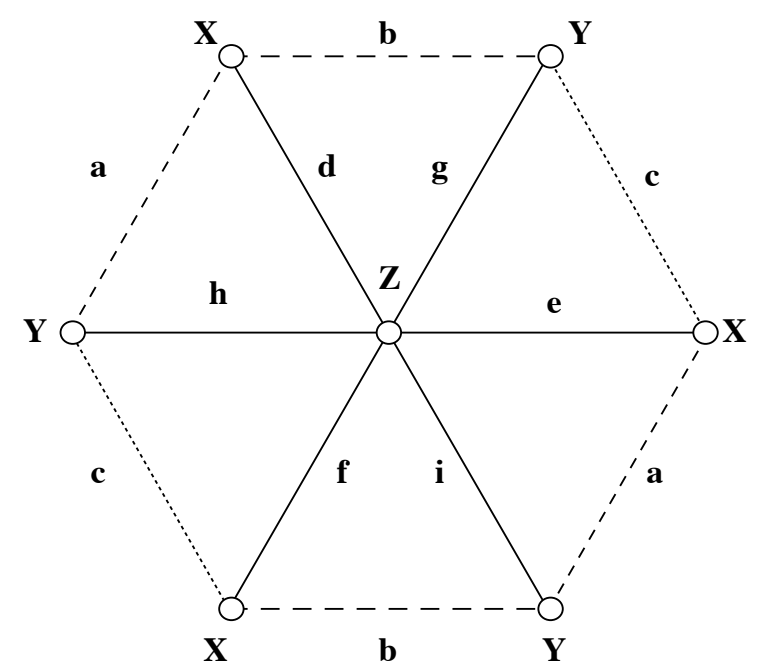

FIG. $5-$ Graph $3 C_{3}$ on the torus, dual to the coffee cup drawing in figure 2.
Theorem 1 can also be used to construct a ctheorem from the same graph $3 \mathrm{C}_{3}$, and it turns out that it is the same Pappus theorem! Indeed, we have already noted that, although it is not immediately obvious, the ctheorems arising from Theorems 1 and 2 are projective duals of one another when viewed in the right way. Pappus is a projectively self-dual configuration (as one sees by switching points and lines), so the two Theorems give the same thing. For Theorem 1, one can take the three points $a, f, g$ to be the vertices of the graph, and the faces to be the remaining points of Pappus: $b, c, d, e, h, i$. Then the edges are the nine lines. For example, the line $a, b, c$ corresponds to the edge between faces $b$ and $c$ having the two vertices $f$ and $g$ as end points.

\section{Further comments}

There are precisely two $8_{4}$ ctheorems of eight points and eight planes in 3-d space over a commutative field, both projectively self-dual; see Glynn (2010). Both have been described figuratively as two tetrahedra that simultaneously inscribe and circumscribe each other. There are five ways of doing this, each way corresponding to a conjugacy class of the symmetric group $S_{4}$, but only two ways give ctheorems. The first $8_{4}$ (Möbius 1828) may be obtained, using either of the Theorems 1 and 2, from the graph $2 \mathrm{C}_{4}$ (the 4-cycle with each edge repeated twice), embedded on the torus with four faces). The second $8_{4}$ may be obtained similarly from the graph $\mathrm{K}_{4}$ plus two disjoint edges also embedded on the torus with four faces; see Glynn (2013) and Alahmadi \& Glynn (unpublished).

Reidemeister configuration in the plane (Reidemeister 1929) is known to be a ctheorem which is algebraically equivalent to the associativity of the additive and multiplicative rings of coordinates for projective planes (Dembowski 1968). Using Theorem 2 its projective dual corresponds to the planar octahedral graph of six vertices, 12 edges, and eight triangular faces with opposite vertices identified or "pinched" together. The process of "pinching" vertices (Archdeacon, Bonnington \& Siran 2014) is a way of obtaining a lower dimensional ctheorem from a higher dimensional one. It corresponds to a projection of the

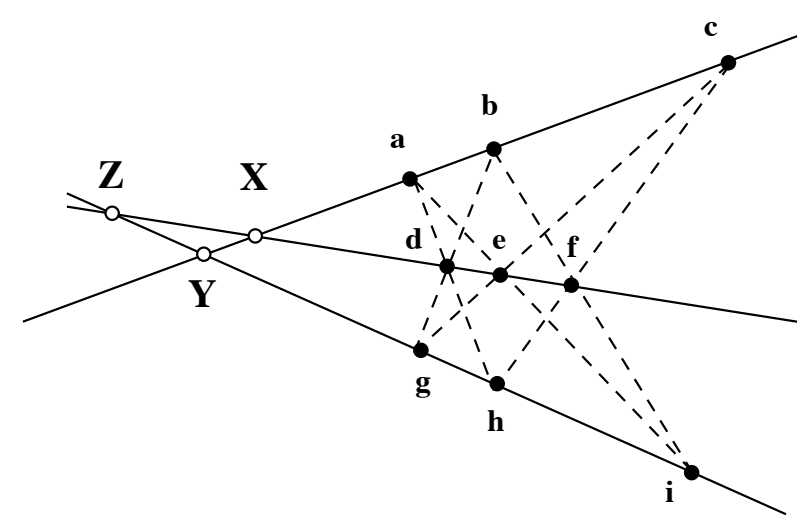

FIG. 6 -Toroidal graph $3 \mathrm{C}_{3}$ (fig. 5), using Theorem 2, implies Pappus ctheorem. 
projective space, and each pinching of a pair of vertices makes the dimension one lower. In this case, five dimensions are projected into two dimensions; see Alahmadi \& Glynn (unpublished).

Consider the planar graph on four vertices: a square with four edges and a diagonal edge. So there are five edges in total and three faces. Then using Theorem 2 one obtains a ctheorem about 3-d space, related to the fact that two lines that intersect are found in a plane. Conversely, in projective geometry coplanar lines must intersect non-trivially.

In axiom systems for projective geometry it is normally assumed a priori that a "line" is a set of points, and then every three of these points are linearly dependent. However, the topology/geometry duality could actually explain this. For example, consider figure 5, which is a hexagon with a central point on the torus. Delete the central point and the six edges through it. There remains a hexagon covering all the torus, folded on itself three times, inducing a graph with two vertices $X$ and $Y$, edges $a, b, c$ and a single face $a b c$. Using Theorem 2 the resulting ctheorem is: if $X, Y, a$, $b, c$ are five points, and $X Y a, X Y b, X Y c$ are three dependent (that is, collinear) sets, then $a b c$ are also collinear.

Interesting papers and a recent book about areas of quantum, strings, and non-commutativity are Twamley (1997), Agagnic (2016), Jones (1987) and David (2015).

\section{ACKNOWLEDGEMENTS}

The authors acknowledge support from King Abdulaziz University, Saudi Arabia.

\section{REFERENCES}

Aganagic, M. 2016: String theory and math: why this marriage may last. Mathematics and dualities of quantum physics. Bulletin of the American Mathematical Society 53: 93-115.

Alahmadi, A.N. \& Glynn, D.G. unpublished: The duality of topology and geometry: why is it So? In submission.

Archdeacon, D., Bonnington, C.P. \& Siran, J. 2014: Regular pinched maps. Australasian Journal of Combinatorics 58: 16-26.

Baker, J. 2014: Cosmology: first light. Nature 509: 276-278.

Bosse, A. 1648 Manière Universelle de M. des Argues pour Pratiquer la Perspective par Petit-pied, Comme le Géomètral: Ensemble les Places et Proportions des Fortes; Foibles Touches, Teintes ou Couleurs. Des-Hayes, Paris.

Carnot, L.M.N. 1803: Geometrie de Position. Paris.

Connes, I. 2004: Symmetries. European Mathematical Society Newsletter (December).

Cowen, R. 2013: Simulations back up theory that Universe is a hologram. Nature News 10th December.

David, F. 2015: The Formalisms of Quantum Mechanics. Lecture Notes in Physics 893: Springer, Heidelberg.

Dembowski, P. 1968: Finite Geometries. Ergebnisse der Math. 44: Springer, Berlin.

Desargues, G. 1639: Brouillon Project d'une Atteinte aux Événemens des Rencontres d'une Cône avec un Plan. (Self-published 50 copies.) English translation, Lloyd Kannenberg 1995: A New Branch of Mathematics. Open Court, Chicago.

Euler, L. 1741: Solutio problematis ad geometriam situs pertinentis. Commentarii academiae scientiarum Petropolitanae 8: 128-140. (Presented to St. Petersburg Academy, 26 August 1735.)

Farmelo, G. 2005: Dirac's hidden geometry. Nature 437 (15 Sept): 323.
Feynman, R.P. 1992: The Character of Physical Law. Penguin, London: $173 \mathrm{pp}$.

Field, J.V. \& Gray J.J. 1987: The Geometrical Work of Girard Desargues. Springer Verlag, New York.

Franzosi, R. \& Guadagnini, E. 1996: Topology and classical geometry in $(2+1)$ gravity. Classical and Quantum Gravity 13: 433-460.

Galison, P. 2000: The suppressed drawing: Paul Dirac's hidden geometry. Representations 72: 145-166. University of California Press.

Gefter, A. 2014a: $M$ is for mystery, how our best theory of everything got its name. New Scientist (19 April). dx.doi. org/10.1155/2013/379074.

Gefter, A. 2014b: Trespassing on Einstein's Lawn. Bantam Press, New York.

Glynn, D.G. 2010: Theorems of points and planes in threedimensional projective space. Journal of the Australian Mathematical Society 88: 75-92.

Glynn, D.G. 2013: A rabbit hole between geometry and topology, ISRN Geometry 2013: Article ID 379074, 9 pp.

Gombrich, E.H. 1977: Art and Illusion A Study in the Psychology of Pictorial Representation. Phaidon Press, Oxford.

Grassmann, H. 1844 Die lineale Ausdehnungslehre. Wiegand, Leipzig: Second edition. 1861.

Hales, T.C. 2001: The honeycomb conjecture. Discrete and Computational Geometry 25: 1-22.

Hamma, A. \& Markopoulou, F. 2011: Background independent condensed matter models for quantum gravity. New Journal of Physics 13:095006.

Hawking, S.W. 1988: A Brief History of Time. Bantam Press, London: $198 \mathrm{pp}$.

Hessenberg, G. 1905: Beweis des desarguesschen Satzes aus dem pascalschen. Mathematische Annalen 61: 161-172.

Hilbert, D. 1902: The Foundations of Geometry. Open Court, Chicago.

IT from Qubit 2015: Simons Collaboration on Quantum Fields, Gravity, and Information, web.stanford.edu/ - phayden/ simons-proposal.pdf. (accessed 19 November 2015).

Jones, J.D.S. 1987: Geometry goes non-commutative. Nature 329: 201.

Klein, F. 1926-27: Vorlesungen über die Entwicklung der Mathematik im 19. Jahrhundert, Two Volumes, Berlin. Reprinted 1967: Chelsea, New York.

Klein, F. 2004: Elementary Mathematics from an Advanced Standpoint: Geometry. Dover, New York. Section on the 1872 Erlanger Programm.

Listing, J.B. 1847: Vorstudien zur Topologie. Göttingen Studien.

Maldacena, J. 1998: The large $\mathrm{N}$ limit of superconformal field theories and supergravity. Advances in Theoretical Mathematical Physics 2: 231-252.

Maldacena, J. 2013: The illusion of gravity. Scientific American 293(5): 56-63 (July).

Möbius, A.F. 1828: Kann von zwei dreiseitigen Pyramiden einejede in Bezug auf die andere um- und eingeschrieben zugleich heissen? Crelles Journal 3: 273-278. In Klein, F. (ed.) 1886: Gesammelte Werke. Leipzig, vol. 1, 439-446.

Mohar, B. \& Thomassen, C. 2001: Graphs on Surfaces. Johns Hopkins University Press, Baltimore.

Nash, C. \& Sen, S. 1983: Topology and Geometry for Physicists. Academic Press, London. Paperback Dover 2011.

Overby, D. 2013: A black hole mystery wrapped in a firewall paradox. New York Times (August 12).

Pappus 320 CE: Collection, Book VII. Royal Library, Alexandria, Egypt.

Plaumann, P. \& Strambach K. 1981: Geometry - Von Staudt's Point of View. Reidel. Proceedings of the NATO Advanced Study Institute, Bad Windsheim, West Germany, 21 July-1 August 1980.

Poincaré, H. 1895: Analysis situs. Journal de l'École Polytechnique (2) 1: 1-123. 
Reidemeister, K. 1929: Topologische Fragen der Differentialgeometrie. V. Gewebe und Gruppen. Mathematische Zeitschrift 29: 427-435.

Riemann, B. 1854: Über die Hypothesen, welche die Geometrie zugrunde liegen (About the hypotheses, which lie at the heart of geometry.) Habilitation Lecture, Göttingen, 10 June. Shared by Dedekind, R., 1868: Abhandlungen der Königlichen Gessellschaft der Wissenschaften zu Göttingen 13: $133-152$.

Robinson, G. de B. 1940: The Foundations of Geometry. University of Toronto Press, Toronto. Book review, 31 January 1942: Nature 149: 125.

Russell, B.A.W. 1897: An Essay on the Foundations of Geometry, Cambridge University Press, Cambridge. Book review, 1897: Nature 56: 417-418.

Stewart, I. 1987: Topology: The hyperbolic phoenix. Nature 328: 16 .

Strogatz, S. 2003: Sync: The Emerging Science of Spontaneous Order. Hyperion Press.

Susskind, L. 1995: The world as a hologram. Journal of Mathematical Physics 36(11): 6377-6396.

Tait, P.G. 1883: Johann Benedict Listing. Nature 27: 316-317 (1 Feb.).

't Hooft, G. 2000: The holographic principle, Opening lecture, SPIN-2000/06. In Zichichi, A. (ed.): Basics and Highlights in Fundamental Physics. The Subnuclear series, vol. 37, World Scientific, 2001, 72-100. hep-th/0003004.

't Hooft, G. 2009: Dimensional reduction in quantum gravity. In Ali, A., Ellis, J. \& Randjbar-Daemi, S. (eds.): Salamfestschrift: A Collection of Talks. World Scientific Series in 20th Century Physics, vol. 4 (World Scientific, 1993), THU-93/26. arXiv:gr-qc/9310026v2, 20 March.

Tutte, W.T. 1959: Matroids and graphs. Transactions of the American Mathematical Society 90: 527-552.

Twamley, J. 1997: Quantum mechanics: statistics given a spin. Nature 389: 127-129.

Veblen, O. \& Young, J.W. 1910, 1917: Projective Geometry, volumes I and II. Ginn \& Co., Boston.

Ver Eecke, P. 1933: Pappus d'Alexandrie, La Collection Mathématique. Oeuvre traduite pour la première fois $\mathrm{du}$ grec en francais, avec une introduction et des notes. Desclée de Brouwer, Brugge/Paris.

Von Staudt, K.G.C. 1847: Geometrie der Lage. F. Korn, Nürnberg.

Whitney, H. 1935: On the abstract properties of linear dependence. American Journal of Mathematics 57: 509-533.

Witten, E. 1998: Anti-de Sitter space and holography. Advances in Theoretical and Mathematical Physics 2: 253-291.

(Accepted 5 July 2016) 